\title{
Introduction to the special issue on the Fourth German Conference on Multiagent System Technologies (MATES)
}

\author{
Klaus Fischer · Ingo Timm • Elisabeth André
}

Published online: 10 May 2008

Springer Science+Business Media, LLC 2008

The articles for this special issue were selected from the submissions to the Fourth German Conference on Multiagent System Technologies (MATES), which took place September 19-20, 2006 in Erfurt in Germany. The driving force behind the MATES series of events is the special interest group for Distributed Artificial Intelligence (SIG-DAI) of the German Informatics Society (Gesellschaft für Informatik E.V.). Founded in 1993 SIG-DAI successfully established the MATES conference series which attracts researchers from all over the world. From 50 submissions 15 were selected as full papers and 8 as short papers. For 4 of the top ranked articles extended versions were accepted for this special issue.

In its character MATES is an application-oriented event and therefore it is not very surprising that the list of articles in this special issue are presenting results that are close to applications at least in a laboratory environment. Although the articles were not meant to directly link to each other, they provide a nice spectrum of different topics that are of interest when it comes to designing agent-based systems or multiagent systems.

Henesey et al. present a multiagent system approach for the evaluation of operational policies in transshipping containers in a simulation environment. In Hahn et al. a metamodel for agent-based systems is presented that supports the development of design tools for such systems. De Jong et al. describe a framework for primary and secondary diagnosis of multiagent plan execution and Bürkle et al. show results of an evaluation of security aspects of mobile agent platforms.

The quality of the articles in this special issue show the scientific strength the MATES conference has gained over the past years.

\author{
K. Fischer $(\varangle)$ \\ DFKI GmbH, 66123 Saarbruecken, Germany \\ e-mail: Klaus.fischer@dfki.de \\ I. Timm \\ TZI-Bremen, Bremen, Germany \\ E. André \\ University of Augsburg, Augsburg, Germany
}

